\title{
The Dutch Interbank Computer Network
}

\section{Eddie MICHIELS}

Heijman and Michiels (H\&M), Lomanlaan 55,

NL-3526 XC Utrecht, The Netherlands

\begin{abstract}
At the end of 1980. a strategic decision was made by the Dutch banks and savings banks to commence the development of a Data Communications Infrastructure (DCI), to be used for a number of forthcoming interbank applications. It was agreed that this new data communications infrastructure should be based on the emerging Reference Model for Open Systems Interconnection (OSI). The first interbank application using the DCI (i.e. urgent money transfers) was introduced in the second quarter of 1985. Other interbank applications, which will also make use of the functions provided by the DCI, are currently being developed.

This paper provides the background to the DCI project, discusses the selection of OSI standards for the network, and gives an overview of the design of the software package, which was developed to support the selected OSI standards.
\end{abstract}

Keywords: OSI, Open Systems Interconnection, OSI software, computer network, interconnection.

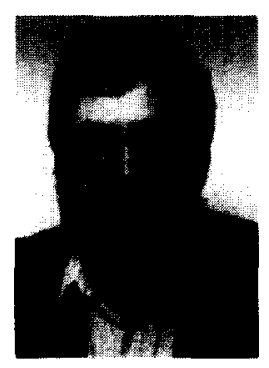

Eddie Michiels joined Electrologica in 1966 to work on the EL-X2 computer project. From 1968 to 1972 , he worked for IBM (systems programming) and Burroughs (data communications) in development laboratories in the USA. Since 1972, as a partner of $H \& M$, he has contributed to numerous consulting and development assignments in the area of data communications and computer networks. He has been an active member of OSI standards committees since 1979 and is currently the editor for the OSI Presentation Layer standards and a liaison representative between SC $21 / \mathrm{WG} 6$ and SC 18 /WG 4 (MOTIS). He is also chairman of the special CEN/CENELEC/ CEPT working group on File Transfer (FTAM) functional standardisation.

North-Holland

Computer Standards \& Interfaces 7 (1988) 127-137

\section{Background to the DCI Project}

\subsection{The Dutch Banking Environment}

The Netherlands Bank Giro Centre (BGC) was founded in 1967 by a consortium of the Dutch banks. The Dutch savings banks joined in 1969 , and it could then be said that BGC represented the entire Dutch banking system (with the exception of the Dutch Post Office bank). The main function of $\mathrm{BGC}$ is centralised (batch) processing of financial bank-to-bank transactions and the provision of information for the clearing between those banks to the Dutch Central Bank.

Transactions originate from a large number of private and public institutions and come in a variety of shapes and forms. In the late seventies. data communications was introduced, allowing the users to transmit batches of transactions to BGC. The IBM BSC $2780 / 3780$ data link protocol was selected as the (de facto) standard for the communications between users and BGC (via switched telephone lines). For security reasons, a pair of encryptor units (based on the DES encryption algorithm) had to be installed on each side of the data link.

In 1980, the banks commissioned a special task force to investigate the feasibility of an interbank data communications network for the exchange of urgent money transfers. These urgent money transfers were still handled between the banks by using telephone or telex and did not involve BGC; the increase in the number of transfers called for automation.

The special task force investigated two entirely different approaches:

1. an ad hoc solution based on de facto standards;

2. the development of a data communications infrastructure (DCI) which should be independent of the application and should be general enough so that is might be used for various interbank applications.

The special task force recommended that the banks should follow the second approach and this recommendation was subsequently approved by the Board of Directors of the BGC. 


\subsection{OSI Standards in the Early Eighties}

In the early eighties, the OSI Reference Model began to take shape. However, the standards for the individual layers were still at an initial stage (with the exception of the three lower layers, where existing standards had been adopted and integrated into OSI). The OSI Transport standards reached the Draft Proposal (DP) status in June 1982, the OSI Session standards reached DP status in May 1983, but underwent significant changes before the Draft International Standard (DIS) status was reached. At that time, not even working documents were available for the OSI Presentation Layer standards.

\subsection{Summary of the DCI Project}

The organisation of the DCI project changed according to the various stages of the project:

\subsubsection{Stage 1: Specification of DCI and Application Protocol}

A DCI standards committee was established by the banks and was assigned the task of selecting appropriate standards and options for the DCI. The members of this committee were the managers of the data communications departments from the participating banks.

At the same time, two committees were established to define the application:

1. a committee representing the end users;

2. a committee with application designers from the participating banks; this committee was assigned the task of developing the application protocol.

\subsubsection{Stage 2: Design and Implementation}

The participating banks were given complete freedom for their implementations of the DCI standards and the application protocol (in the spirit of OSI). However, it was recognised that it would be impossible for the small and very small banks to develop their own implementations of the DCI standards (at that time, no OSI products were available). Since it was essential to the success of the project that the smaller banks should participate, it was decided that BGC would implement two types of end systems to serve these participants:
1. for the small banks: a (cheap) minicomputer system, to be installed at the bank's premises, with a number of end user terminals attached;

2. for the very small banks: a computer system installed at the BGC site, to which a number of remote IBM-compatible personal computers or 3270 terminals could be attached, as well as telex equipment (which most of the banks already had installed).

The larger banks decided to develop their own implementations.

At the time, most of these banks were using IBM mainframes and they were also in the process of deploying their own (intrabank) network, some of which were not based on SNA concepts and products.

BGC had large Burroughs mainframes installed, as well as some smaller IBM mainframes. The Name and Address file (20 million accounts) was implemented on the Burroughs system and since access to this file was essential for the application, it was decided that the Burroughs mainframes should be connected to the $\mathrm{DCl}$ network.

\subsubsection{Stage 3: Testing of DCI Implementations}

Because it was not feasible to synchronize the developments taking place in all the participating banks, there was a certain risk that the connection of a new participant to the running DCI network might disturb its correct working. Therefore, it was decided that BGC should develop test tools, so that a new DCI implementation could be verified to conform to the DCI standards, before connection to the running network would be allowed.

\section{Selection of Standards for the DCI}

After some preliminary discussions, it was agreed that the DCI standards should follow the OSI standards as closely as possible. Given the status of these OSI standards in the 1982-1983 period, the DCI standards committee took a relatively long time before reaching consensus on the specific standards and to be included in the DCI. In some cases, dramatic changes in the OSI standardisation process made it necessary for the committee to annul previously made agreements and to start all over again. 
The following issues had to be resolved by the DCI standards committee:

1. It was decided to use the Dutch public packet switching network (Datanet-1) to provide the services of the OSI Network Layer. However, at the time no (draft) standards or working documents were available that specified the mapping of the X.25 protocol elements onto the OSI Network Service Definition. Therefore, the committee had to define those mappings.

2. Because of the tariff structure imposed by the Dutch PTT, PVCs (permanent virtual circuits) had to be used. It was discovered that the OSI Network and Transport Layer standards did not harmonize very well with the use of permanent virtual circuits (note: ISO/TC 97/SC 6 has recently decided to resolve this issue).

3. It was decided not to use the expedited and delivery confirmation options of the OSI Network Service because they were not required by the transport protocol standard.

4. In order to isolate the upper layers from address changes necessary for reconfiguration and back-up purposes, it was decided to use address mapping tables at the network level. A provision for multiple subnetworks was also incorporated in these network address tables (to allow for future extensions of the DCI with other subnetwork types).

5. A selection had to be made from 5 different transport protocol classes. Classes 0 and 1 were rejected because the banks did not want to become dependent on the Dutch PTT for multiplexing (i.e. assigning $X .25$ logical channel numbers). Class 4 was rejected because it was considered to be too complex and not really necessary when using a reliable X. 25 network. Class 3 was selected because it was considered essential that the Transport Layer should be able to recover from X.25 network resets and restarts. The choice for class 3 also implied that class 2 had to be implemented (OSI transport protocol conformance requirement).

6. It was decided that the "no explicit flow control" option of transport protocol class 2 should be supported by a full-blown DCI implementation (although it is only a non-mandatory option in the OSI standard), to allow for the interworking with less sophisticated DCI implementations.

7. A requirement for the use of extended TPDU formats was not identified and therefore not in- cluded in the DCI specifications.

8. The transport expedited option was selected (because the final requirements of the session protocol were not known at the time this decision had to be made).

9. The OSI transport protocol did not allow the multiplexing of transport connections to be initiated by different sides (the NCMS addendum to the transport standards was not available at the time). It was decided that this restriction was too severe when using X.25 PVCs and that the DCI should allow this type of multiplexing.

10. The messages exchange by the network had to be secured. No OSI security standards were available; therefore, it was agreed to to provide for (optional) encryption at the transport level. It was decided to use the transport protocol security parameter for the exchange of encryption working keys (encrypted with a master key) during the establishment of the transport connection. DEA (DES) was selected as the encryption algorithm.

11. A transport suffix was selected for addressing at the transport level.

12. A DCI transport protocol document was produced, containing only the clauses from the ISO document that were relevant to the selected transport protocol classes and options.

13. It was decided that the store-and-forward concept (then being defined by CCITT in its draft $X . m h s$ recommendations) was not suitable for interbank applications, for the following reasons:

- delivery notification was (and still is!) an ill-defined concept; therefore, the application protocol would need to contain commitment mechanisms already provided by the session layer;

- message security is difficult to achieve in a store-and-forward messaging environment;

- the method is inherently unsuitable for bulk data transfer because of the unnecessary requirement to store the data in its entirety before forwarding it.

14. After the DCI standards committee had reached an agreement to use an interim Session Layer standard (i.e. ECMA-75), a dramatic change in the OSI standards arena forced the committee to reconsider this issue. It was then decided to follow the new OSI direction (i.e. a session protocol based on the synthesis of the ECMA-75 standard and the CCITT recommendation S.62 for Teletex).

15. The selection of a session service subset (i.e. 
a specific combination of session functional units) and session options proved to be a difficult task. The banks had to commit themselves for each financial transaction and no transactions should be lost or duplicated; therefore, synchronisation and resynchronisation were essential. These functions could be provided by either the BSS or BAS session subsets. For performance reasons, the (half-duplex) BSS subset was selected (including the extended concatenation option).

16. The use of the transport expedited option (extended control) was selected.

17. A session suffix was selected for addressing at the session level.

18. A DCI Session Service and Session Protocol document was produced, which contained only the information relevant to the implementation of the selected session subset and session options. Because of the structure of the ISO session standards, this was a rather difficult task.

19. No OSI Presentation Layer standards were available; therefore, the committee had to develop a (simple) interim standard for this layer. The nature of the messages exchanged required the inclusion of a compression and decompression algorithm in this interim standard.

20. No OSI standards were available for an abstract syntax notation and the DCI standards committee therefore decided to define a rudimentary abstract syntax notation, which included only a few simple data types (character strings, decimals, and octet strings). A corresponding transfer syntax (encoding rules) was also defined by the DCI standards committee (including the compression and decompression algorithm).

\section{Application Protocol Specification}

In a banking environment, application standards developed by ISO/TC 68/SC 5/WG 4 (Applications of OSI in Banking) should be followed whenever possible. However, at the time no such standards or working drafts were available and the committee had to start from scratch.

The lay-out of the messages to be exchanged was agreed upon and then specified using the abstract syntax notation defined for the DCI.

A simple application management protocol was defined (to enable re-establishment of connections after the occurrence of a failure signaled by the
DCI). One of the problems encountered here was the fact that the OSI standards did not contain a very useful distinction between transient and permanent errors.

\section{Implementation of the DCI Standards}

Three of the major banks made the decision to implement the application using IBM's IMS data base management system. It was therefore agreed that BGC should also develop a gateway between SNA/IMS and OSI/DCI protocols (see Fig. 1). A global design was made for a transparent SNA/OSI gateway implementation, but the characteristics of the IMS SNA protocols (LU type P) imposed many severe constraints. It was therefore

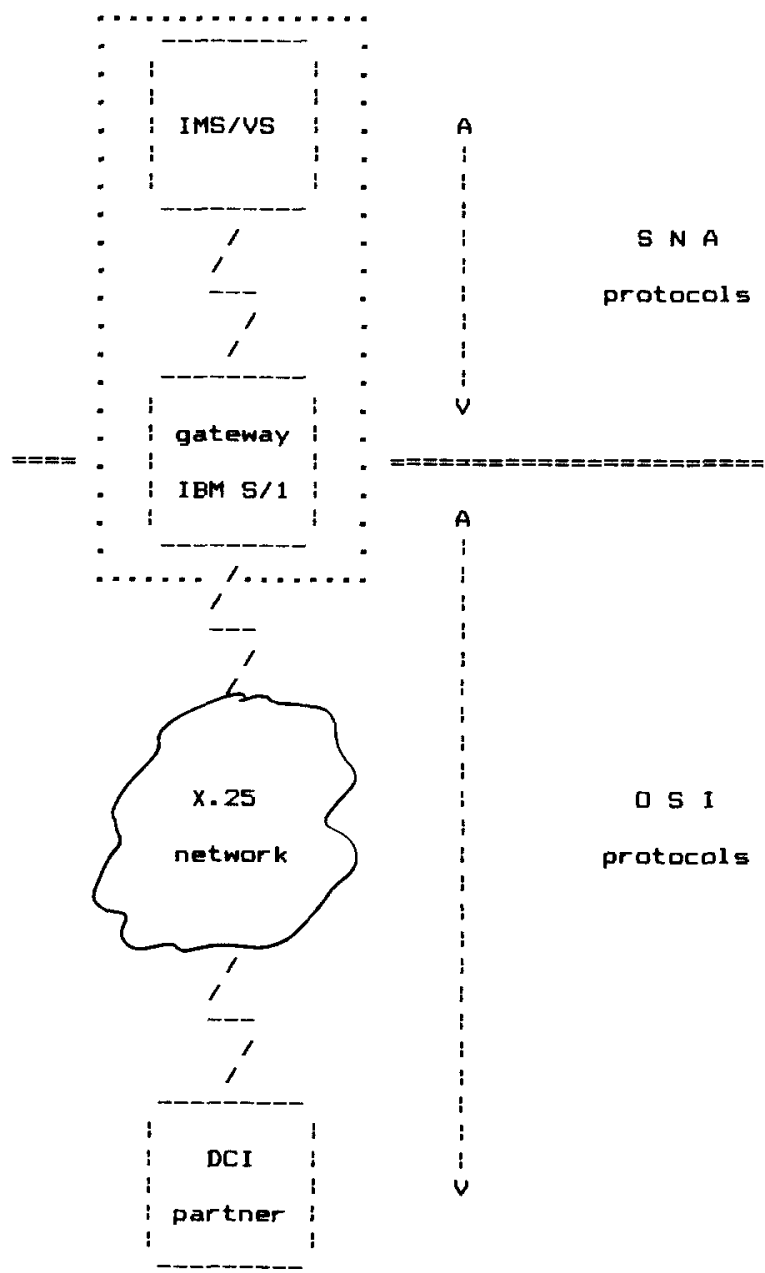

Fig. 1. OSI/SNA Gateway. 
decided that the gateway should only support predefined IMS transaction types (with complete message transparency), because this would significantly reduce the gateway implementation effort.

As a result of the decision, BGC now had to provide DCI implementations for the following end systems (see Fig. 2):

1. its own complex of Burroughs mainframe computers:

2. a computer system at BGC to which the very small banks could connect, using PCs or 3270 terminals (via leased or switched telephone lines) or telex equipment;
3. a number of small systems, to be installed at the smaller banks (with a limited number of terminals attached);

4. a number of OSI/DCI-SNA/IMS gateways to be installed at some of the major banks.

One of the major banks decided to implement the DCI protocols on a Tandem computer system.

BGC decided to select a computer system with a wide performance range (including a small and cheap starting configuration) and equipped with proven X.25 and SNA (LU type P) software. The IBM Series 11 computer was chosen and the OSI (DCI) protocols were implemented on this com-

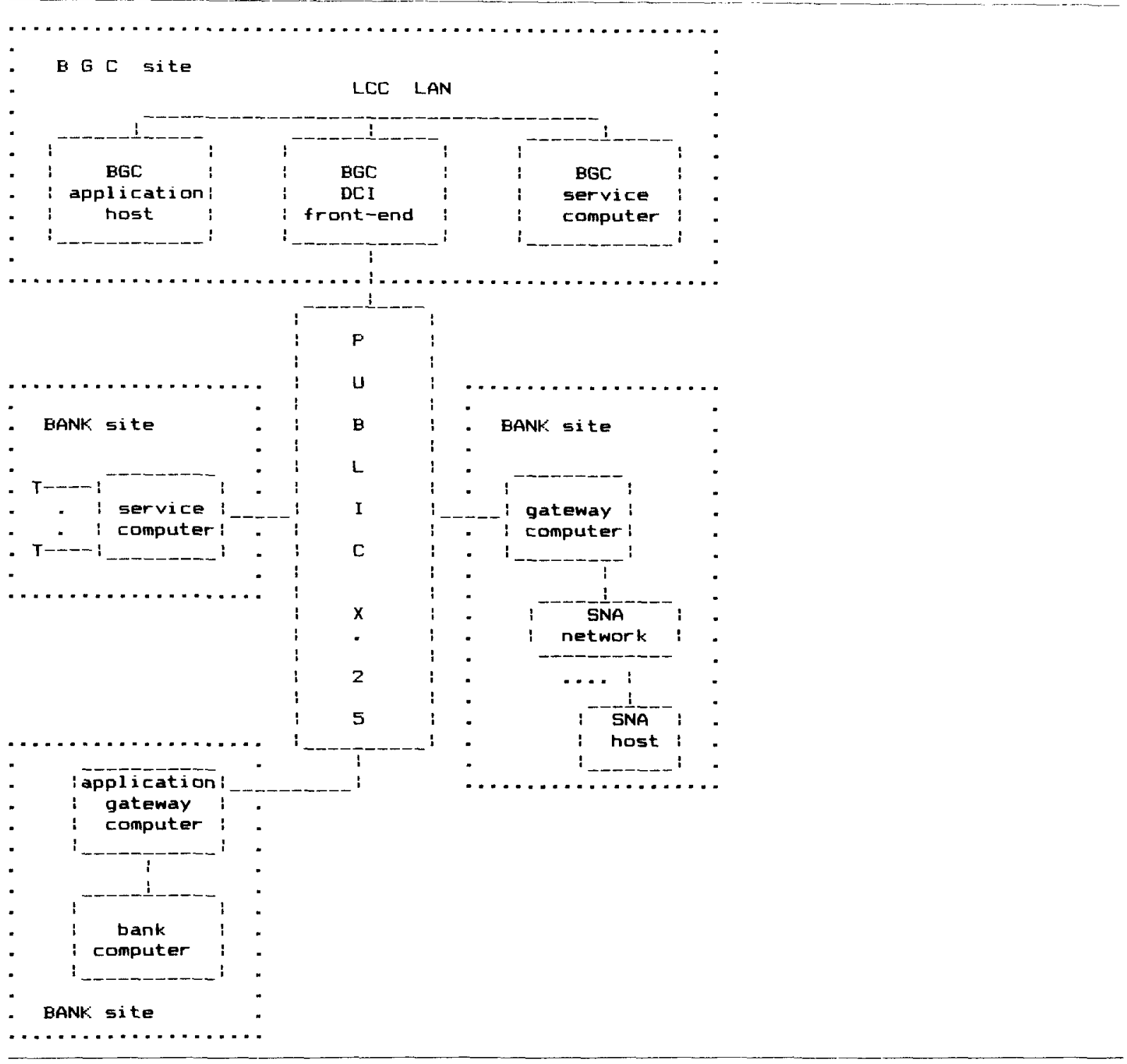

Fig. 2. BGC Implementations. 


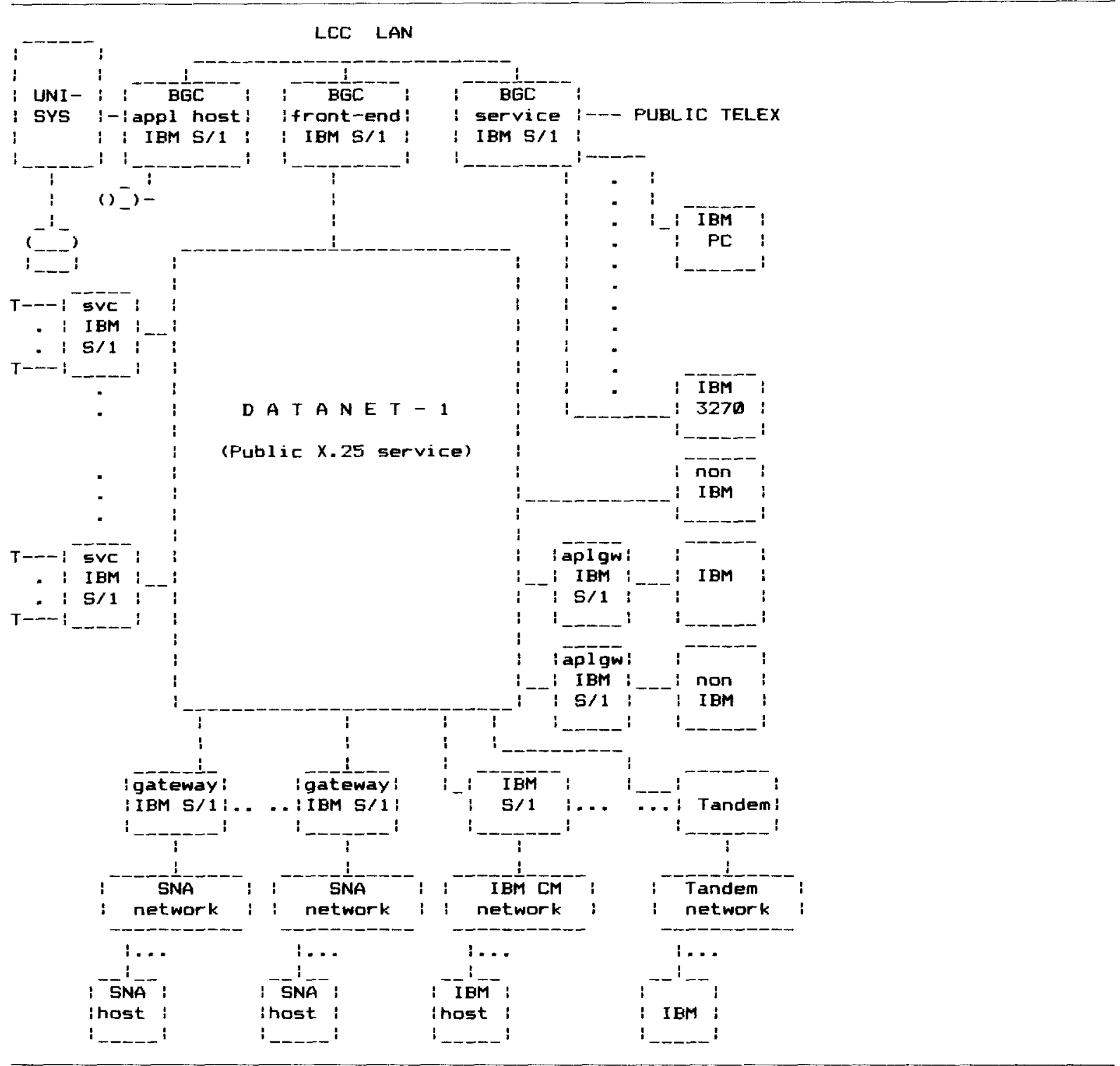

Fig. 3. DCI Overview.

puter system in such a way that the resulting DCI software could be configured for use in a variety of IBM Series/1 configurations (single systems, clustered systems, distributed systems, etc.).

For an overview of the DCI network configuration, see Fig. 3.

The OSI standards proved to be very difficult to understand and to implement for anyone who had not been involved in the standards making process. This was especially true for the rather complicated session protocol. The DCI implementation teams were therefore generating lots of questions related to the session standards. In order to provide quick and accurate answers to these questions, $\mathrm{H} \& \mathrm{M}$ developed a session simulator program (running on a $\mathrm{PC}$ ), which could be used to print time-sequence diagrams and to display the state and variables of the session protocol machine. 


\section{IBM Series / 1 DCI Software Design}

\subsection{Network Layer}

IBM's Series/1 Packet Network Support software provides the necessary support for the connection to the Dutch Datanet-1 public packetswitching network. This software was enhanced, to enable the use of other subnetwork types (e.g. leased lines) in the future. In addition to this, software was developed which allowed for the use of memory queues (instead of X.25 virtual cir- cuits) for the interworking - via a common DCI front-end - of DCI end-systems installed at the same physical location.

Configuration management sofware was also developed for the DCI network layer (including support for address mapping tables).

\subsection{Transport Layer}

It was decided that the DCI software package should contain only one bufferpool (to store data in transit) and that this bufferpool should be

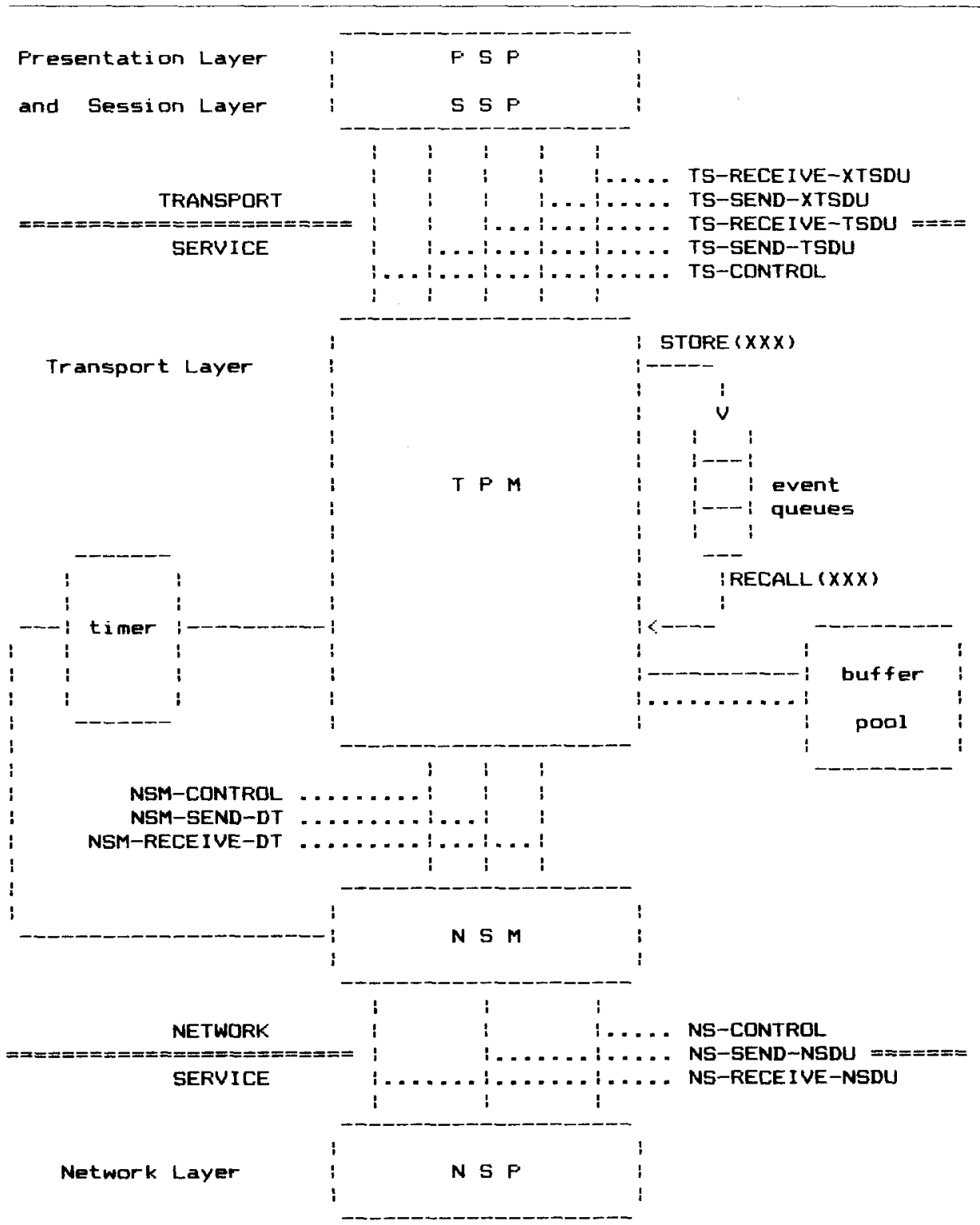

Fig. 4. DCI Design Overview. 
located at the transport level (because the transport protocol provides end-to-end flow control). The bufferpool normally contains TSDU segments and the segment size reflects the selected maximum TPDU and NSDU/NPDU sizes, in such a way that concatenation of $\mathrm{AK}$ and DT TPDUs is always possible.

The Transport Layer is divided into two modules (see Fig. 4):

1. Transport Protocol Machine (TPM) module; and

2. Network Service Mapping (NSM) module.

The TPM module consists of four submodules (corresponding to the connection establishment, data transfer, resynchronisation, and release phases of the transport protocol). The data transfer submodule itself consists of four other submodules that run in parallel (send, receive, send

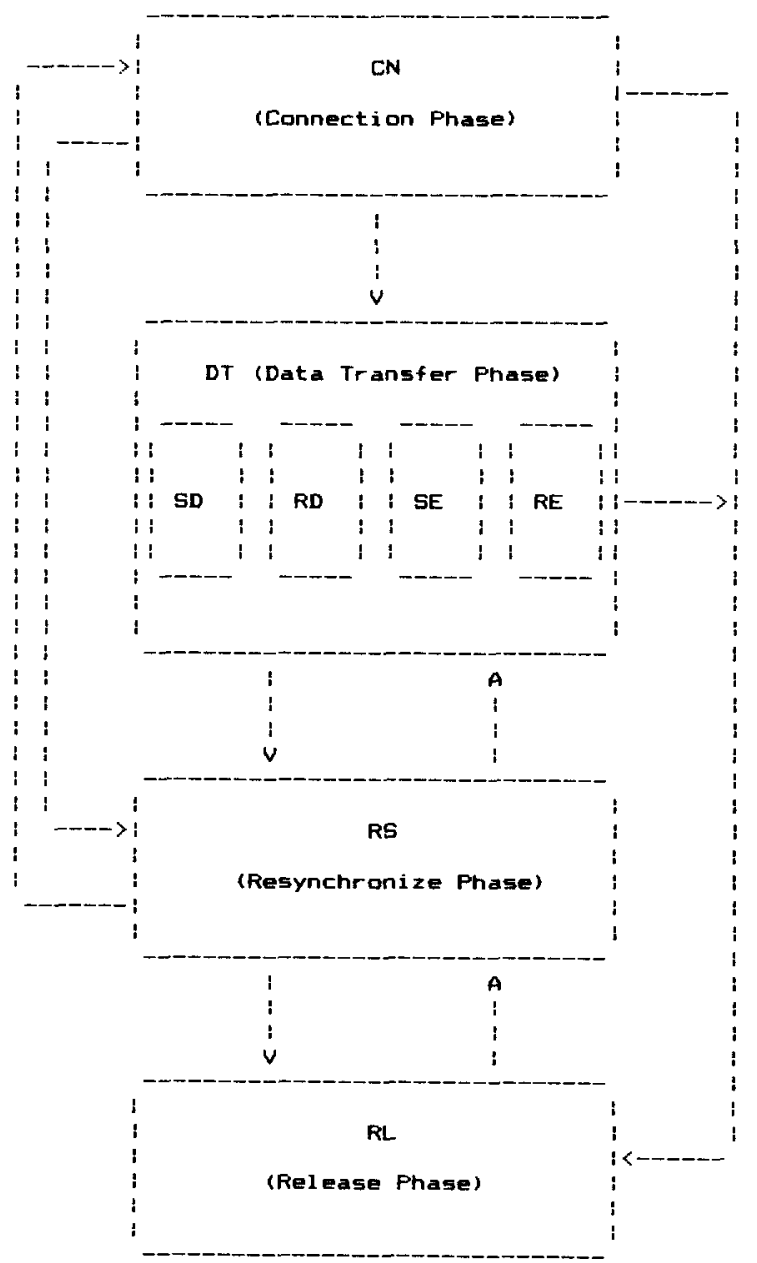

Fig. 5. TPM Overview. expedited, and receive expedited). See Fig. 5.

The NSM module implements those transport layer functions that apply to more than one transport connection (multiplexing, concatenation of TPDUs, network resets, etc.).

The DCI Transport Layer has been designed in such a way that future incorporation of transport protocol classes 0 and 1 will be easy to accomplish.

\subsection{Session Layer}

The DCI Session layer implementation does not contain a message storage facility (see above). Each session event (either an SS-user request/response or an incoming SPDU) is processed immediately by the SPM (Session Protocol Machine) module.

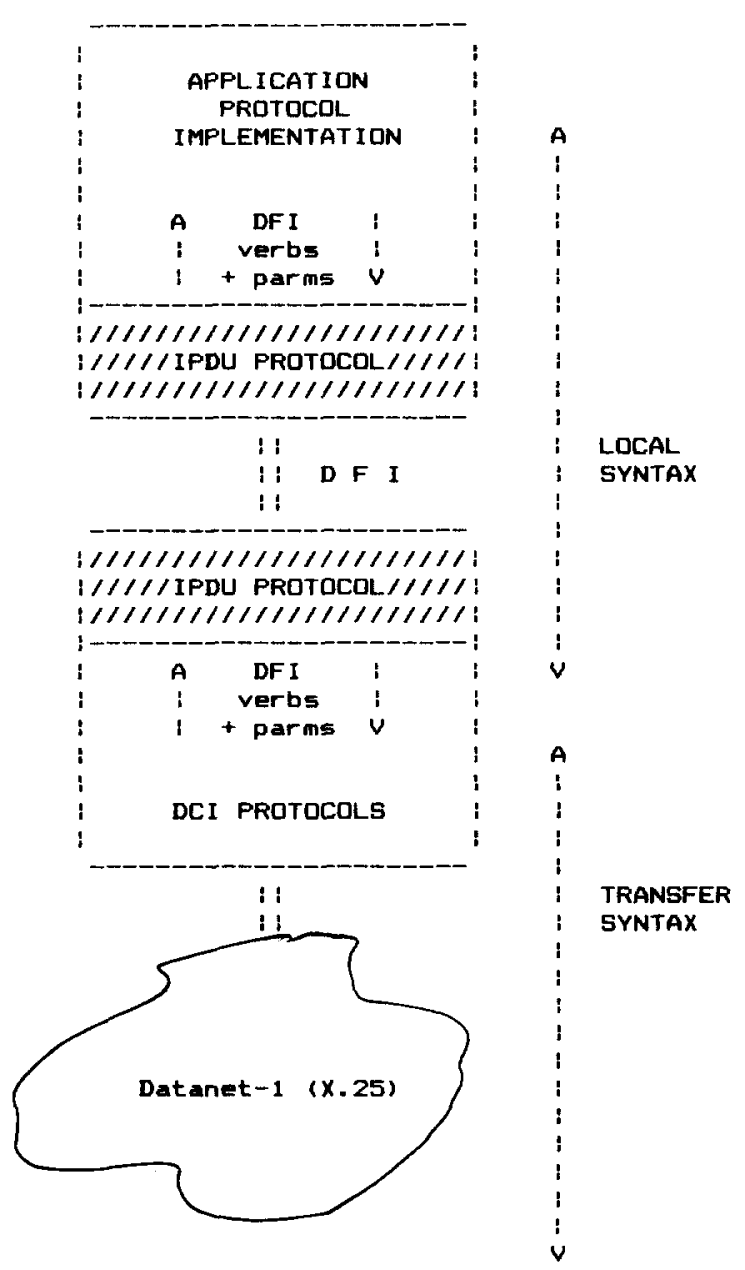

Fig. 6. DCI Front-End Interface. 
The DCI Session Layer design reflects the structure of the OSI session standard state/event tables; therefore, future extension with other functional units (e.g. those required for the BAS subset) will be straightforward.

\subsection{Presentation Layer}

DCI Presentation Layer functions have been implemented as subroutines called by the DCI Session Layer implementation (or vice versa). The design is such that migration to the OSI Presentation Layer standards will be easy (the notion of local and transfer syntax selection has already been incorporated in the DCI software, although only one of each of these syntaxes is currently supported).

\subsection{Front-End Interface (DFI)}

An interface between the DCI (front-end) software (which implements the OSI standards selected for the DCI) and the application programs has been defined and well-documented. A number of verbs and parameters provide for the exchange of information between the applications and the DCI front-end (see Fig. 6).

The physical transport of these DFI (DCI Front-end Interface) verbs can be realized by different means:

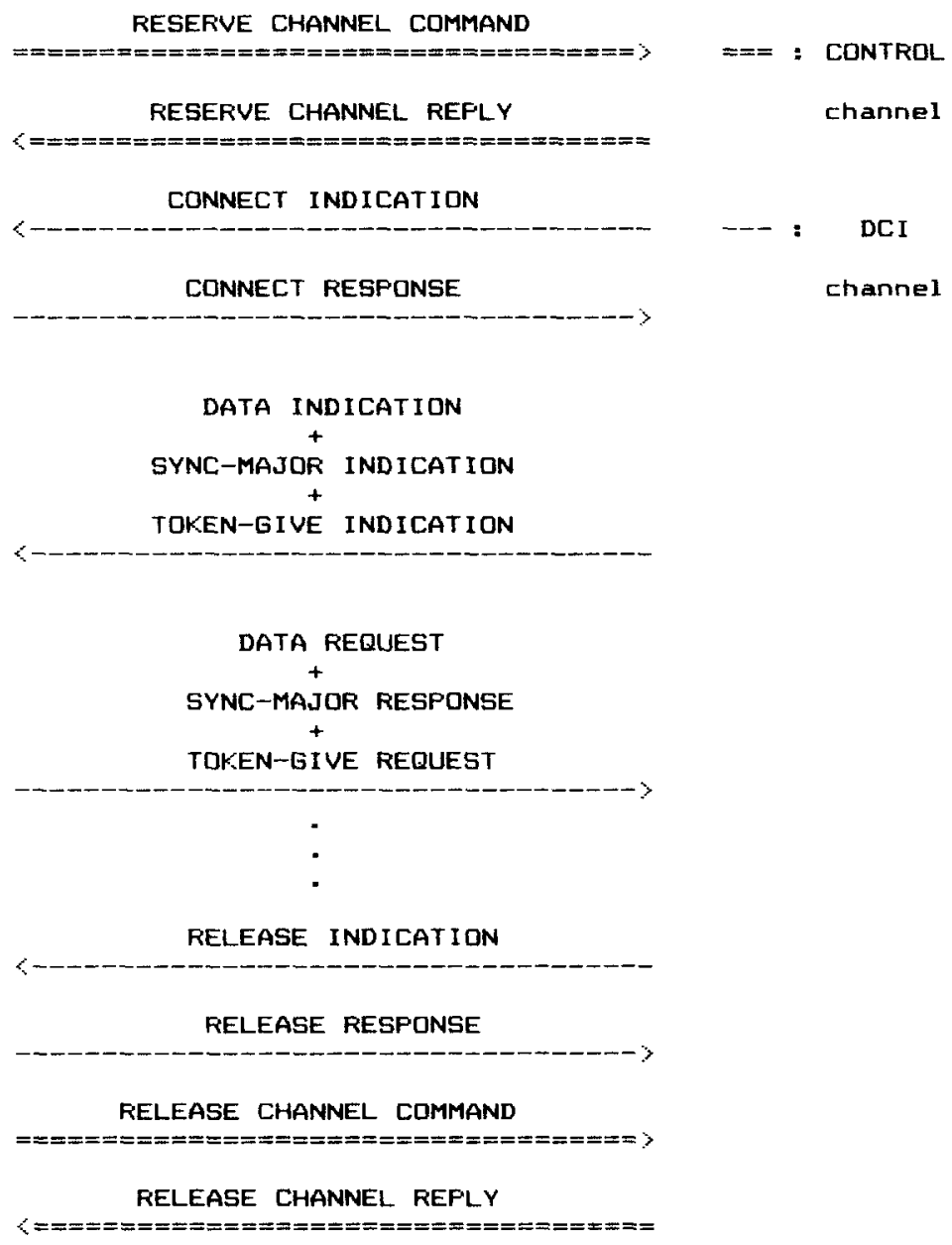

Fig. 7. Example of Usage of DFI Verbs. 
- memory queues;

- disk queues;

- local area networks;

- other communication links (X.25 virtual circuits, HDLC links, IBM BSC links, IBM SNA links, etc.).

Currently, all communication facilities offered by the IBM Series/1 Communications manager (CM) software package are supported.

Two types of DFI verbs have been defined:

1. DCI verbs, which correspond to the OSI session (presentation) service primitives;

2. CONTROL verbs, which are used for local (configuration) management purposes.
Fig. 7 illustrates the flow of DFI verbs over the interface between an application and the DCI package. The exchange of DFI verbs follows the rules specified by the IPDU (Interface Protocol data Unit) protocol (see Fig. 8). This protocol provides the following features:

- concatenation of DFI verbs (concatenations allowed by the OSI session protocol);

- fragmentation of (possibly concatenated) DFI verbs;

- back-pressure (in both directions);

- normal and urgent IPDU transfers.

The communication links and associated communication protocols used for the realisation of

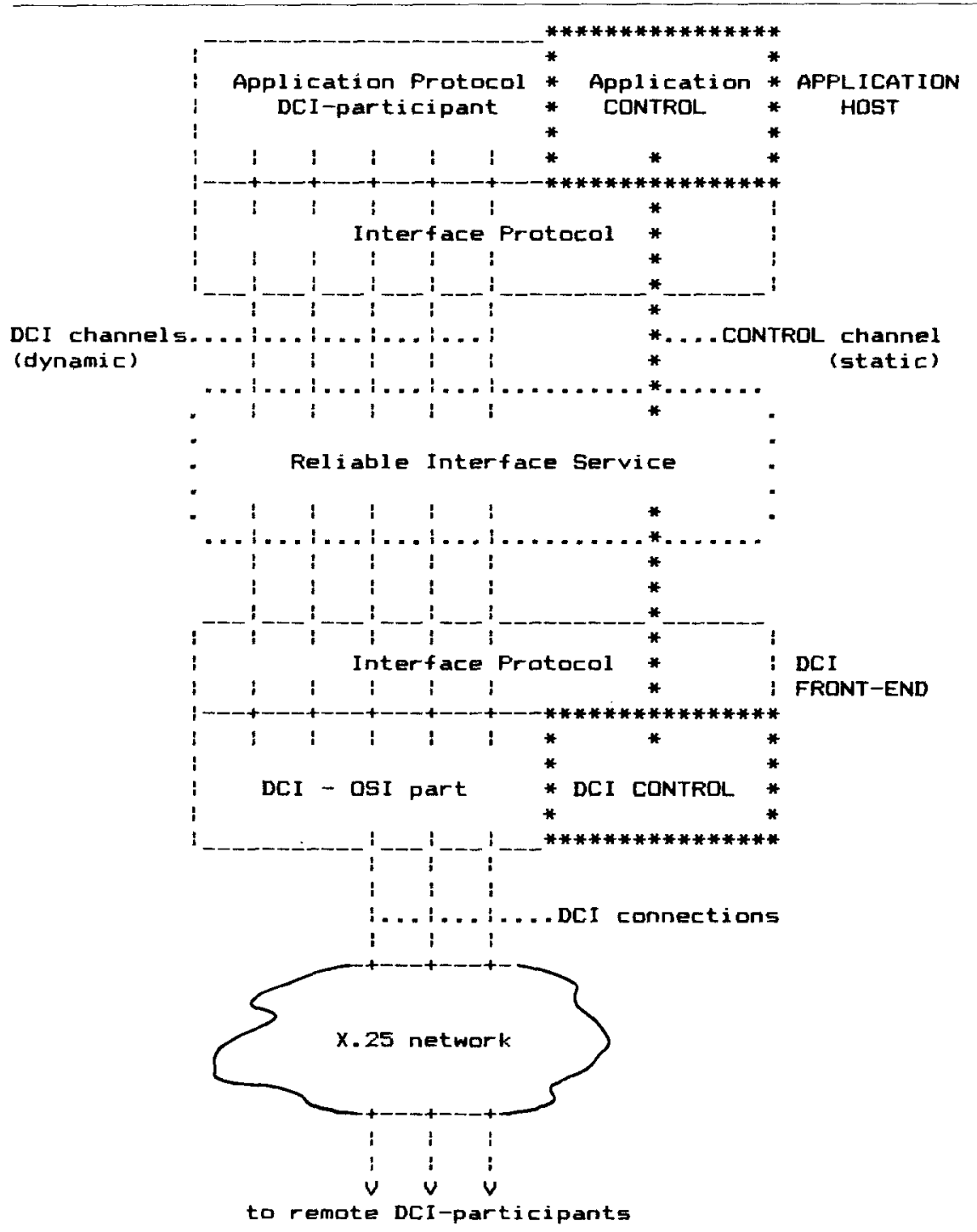

Fig. 8. DCI Front-End Interface (DFI). 
the DCI Front-end interface (DFI) must provide a very reliable transfer service since the IPDU protocol itself does not include any recovery mechanism.

\section{Testing of DCI Implementations}

BGC developed test tools (see Fig. 9), which were first used to test its own DCI implementations. The OSI session simulator was used for the preparation of test scenarios. The test tools were subsequently used to test DCI implementations from other DCI development teams. The OSI session simulator turned out to be a valuable tool for arbitration during these tests. Recently, the test tools were used to test an OSI software package of a well-known computer manufacturer, using international X.25 virtual circuits.

\section{Conclusion}

In a heterogeneous environment, in which many organisations are involved, OSI can prove to be the only workable solution (because it may not be possible to agree on the use of standards developed by a particular computer manufacturer). This may even be true for what could otherwise be perceived as a homogeneous environment. For example: a group of IBM users may not be able to reach an agreement to use SNA protocols, because some of these IBM users may have non-SNA networks installed.

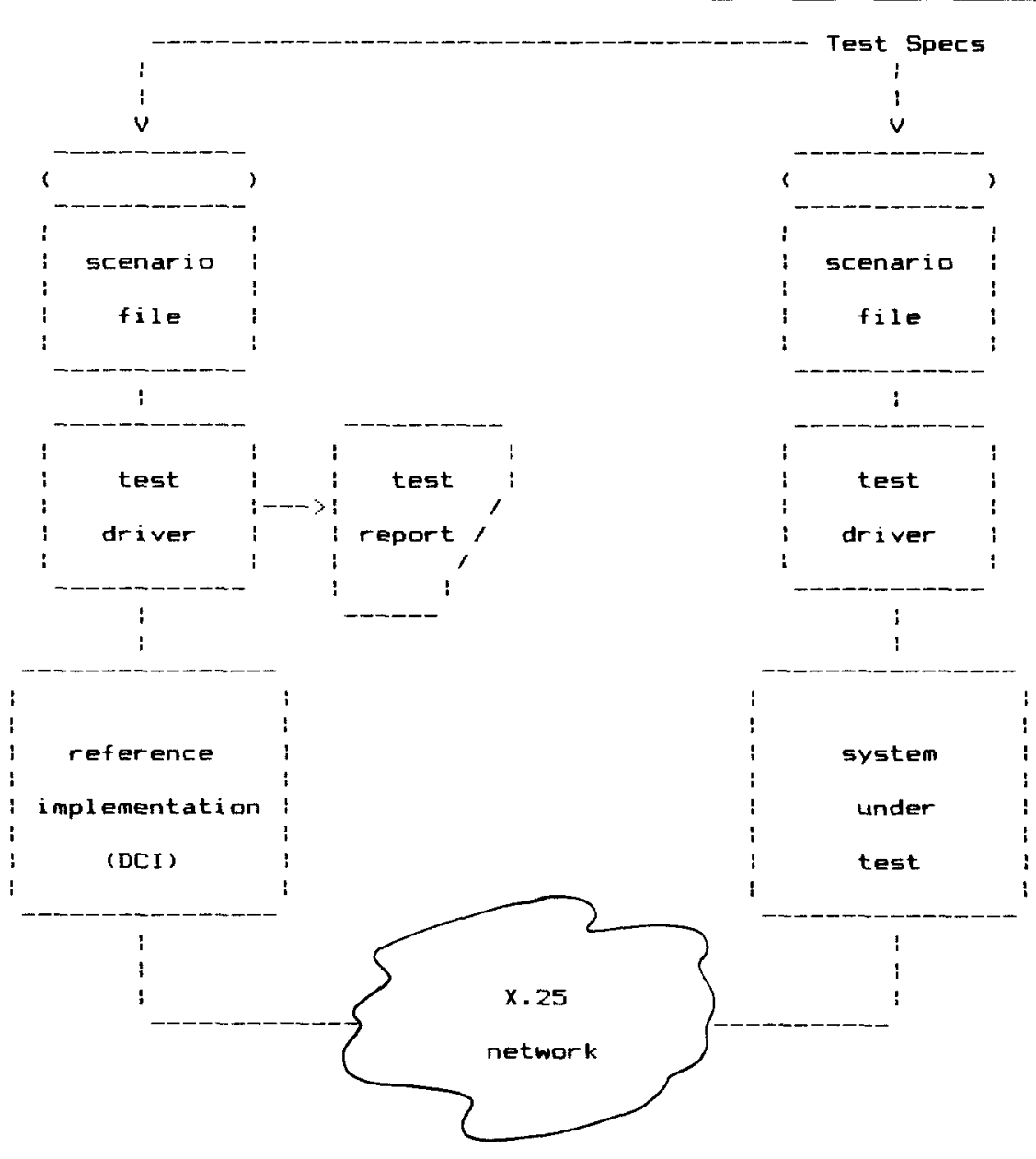

Fig. 9. Testing Methodology. 